\title{
MASTOFAUNA DE LA RESERVA DE LA BIOSFERA "LA ENCRUCIJADA", CHIAPAS
}

\author{
EDUARDOESPINOZA ${ }^{1}$, EPIGMENIO CRUZ ${ }^{2}$, HELDA KRAMSKY ${ }^{1}$ E \\ IGNACIOSANCHEZ ${ }^{3}$
}

${ }^{1}$ El Colegio de la Frontera Sur, Carr. Panamericana y Periférico Sur, San Cristóbal de Las Casas, 29230 Chiapas.e-mail: emedinilla@sclc.ecosur.mx

${ }^{2}$ Instituto de Historia Natural y Ecología del Estado de Chiapas. Calz. Cerro Hueco s/n Tuxtla Gutiérrez, 29000 Chiapas.e-mail:Cruz5910@prodigy.net.mx

${ }^{3}$ Fundación Arqueológica Nuevo Mundo. Orquídea 16 Barrio de María Auxiliadora 29230 San Cristóbal de las Casas, Chiapas.e-mail: jnacho_sanchez@hotmail.com

\begin{abstract}
Resumen: Se presenta una lista de mamíferos de la Reserva de la Biosfera “La Encrucijada”. Ésta área protegida está situada en la franja de manglar de la zona costera del Estado de Chiapas. Un total de 144,868 ha cubren 8 Municipios, Pijijiapan, Mapastepec, Acapetahua, Villa Comaltitlán, Huixtla, Huehuetán, Mazatán. En muestreos periódicos que se llevaron a cabo durante varios años, fueron encontradas 69 especies y 23 familias en 8 ordenes. "La Encrucijada” representa el 15\% total de especies de mamíferos en México (477) y el 33.8\% para el estado de Chiapas (204).
\end{abstract}

Palabras clave: Reserva Biosfera, Encrucijada, Conservación, Mamíferos.

Abstract: A mammal checklist of the Reserva de la Biosfera "La Encrucijada" is presented. This protected area is located in the pacific coast of the State of Chiapas, in the mangroove zone. A total of 144, 868 ha are covered in eight municipalities (Pijijiapan, Mapastepec, Acapetahua, Villa Comaltitlán, Huixtla, Huehuetán, Mazatán). In a few years of periodical sampling, 69 species and 23 families in 8 orders, were found. "La Encrucijada” represents 15\% of the total mammalian species in Mexico and the $33.8 \%$ of the state of Chiapas.

Key words: Biosfera Reserve, Encrucijada, Conservation, Mammals.

\section{INTRODUCCIÓN}

La Reserva de la Biosfera "La Encrucijada” (REBIEN) se localiza en la Planicie Costera del Pacífico del Estado de Chiapas (Figura 1). Fue decretada en mayo de 1972 como Área Natural y Típica del Estado de Chiapas, “Tipo Ecológico Manglar Zapotón”, con una superficie de 2,500 hectáreas. Debido a su importancia como 
sistema lagunar, fue decretada como Reserva de la Biosfera “La Encrucijada”, por un decreto federal, publicado en Junio de 1995, localizada entre los $14^{\circ} 43^{\prime}$ y $15^{\circ} 40^{\prime}$ latitud Norte y $92^{\circ} 26^{\prime}$ y $93^{\circ} 20^{\prime}$ longitud Oeste. Actualmente, la reserva cuenta con una superficie de 144,868 ha y se localiza en los municipios de: Pijijiapan, Mapastepec, Acapetahua, Villa Comaltitlán, Huixtla, Huehuetán y Mazatán.

El clima de la región de la costa es del tipo Am (w) cálido-húmedo, con abundantes lluvias en verano. La precipitación mínima anual es de 2,500 mm, y la máxima es de 3,000 mm, repartidos entre 100 y 200 días lluviosos al año. La temporada de lluvias comienza en el mes de mayo y se extiende hasta noviembre, presentándose la sequía intraestival de julio a agosto; el resto del año es seco o con lluvias ocasionales en febrero o marzo. La temperatura es constante todo el año, siendo mayor de $22^{\circ} \mathrm{C}$ (García, 1973). Se encuentran nueve tipos de vegetación, entre los que destaca por su abundancia y cobertura, el manglar, zapotonal, tular, popal, selva mediana subperenifolia, selva baja caducifolia, dunas costeras, vegetación flotante y subacuática y palmares.

La REBIEN, es la única área en el estado que protege las especies de flora y fauna existentes en el Sistema de Humedales de la Costa de Chiapas. Además, está catalogada como la más importante de la Costa del Pacífico Americano, debido a su extensión, estructura y productividad (Contreras, 1988 y 1993).

Es factible pensar que ésta región, se ha constituido como un refugio obligado para alojar a las especies de mamíferos que ahí se distribuyen. La pérdida de hábitat en la costa de Chiapas, debido principalmente a las actividades de agricultura y ganadería, han modificado los patrones de biodiversidad. En los últimos 50 años se ha reducido de forma alarmante la presencia de fauna y flora silvestre en la planicie costera de Chiapas, formando una delgada franja, que por su infranqueabilidad permite la sobrevivencia de poblaciones animales.

\section{MÉTODOS}

Se llevaron a cabo ocho viajes de campo con duración de siete días cada uno, durante el periodo de abril de 1994 a mayo de 1996. Para la colecta de mamíferos no voladores se colocaron 100 trampas tipo Sherman y 20 Tomahawk durante un promedio de cinco noches por viaje. Para la captura de murciélagos se instalaron cinco redes de niebla entre las 18:00 y las 02:00 durante un periodo de seis noches por viaje. Fue utilizado también un rifle calibre .22 para la colecta de ejemplares de tamaño medio. Por las noches se hicieron recorridos nocturnos con lámparas sordas de alta potencia en las islas y a lo largo del Río Huixtla con la finalidad de registrar animales con hábitos nocturnos. También fueron tomadas huellas con moldes de yeso y se obtuvieron restos óseos encontrados durante los recorridos nocturnos por la zona. 
Los ejemplares obtenidos fueron depositados en la Colección Zoológica Regional del Instituto de Historia Natural del Estado de Chiapas, y algunos fueron donados al Museo de Zoología de la Facultad de Ciencias de la Universidad Nacional Autónoma de México.

La determinación taxonómica de los ejemplares colectados se llevó a cabo siguiendo las claves de Álvarez et al., (1994), Hall (1981), Medellín et al., (1997), Reid (1997) y Villa (1966). Fue solicitada la información de las bases de datos y catálogos de 36 museos nacionales y extranjeros que pudieran tener en su acervo ejemplares del estado de Chiapas, de los cuales sólo nueve tuvieron información de la REBIEN (Apéndice 1).

La distribución y estado de conservación de las especies se estableció de acuerdo a la Unión Internacional para la Conservación de la Naturaleza y Recursos Naturales (IUCN por sus siglas en inglés), la Convención Internacional de Tráfico de especies de Flora y Fauna Silvestres (CITES) y por la Secretaria de Medio Ambiente y Recursos Naturales (Norma Oficial Mexicana SEMARNAT NOM-059-ECOL-2002, Protección Ambiental-Especies Nativas de México de Flora y Fauna SilvestresCategorías de Riesgo y Especificaciones para su Inclusión, Exclusión o Cambio-Lista de Especies en Riesgo).
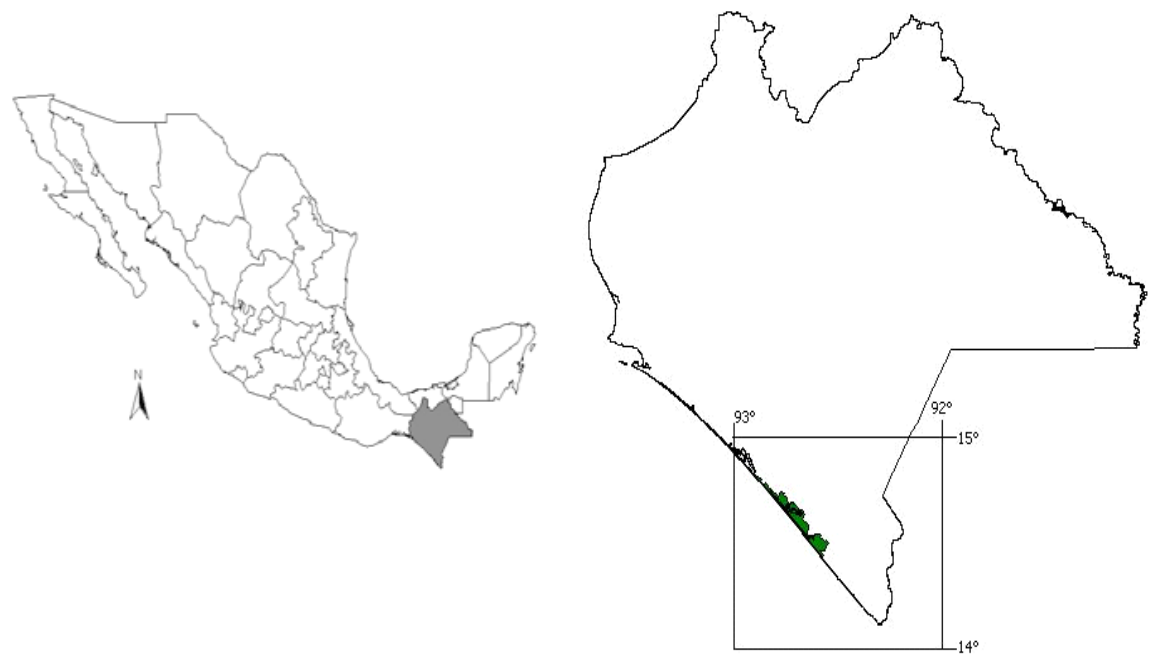

Figura 1. Ubicación de la Reserva de la Biosfera La Encrucijada (REBIEN), en la costa de Chiapas. 


\section{RESULTADOS Y DISCUSIÓN}

La mastofauna de la REBIEN está integrada por 8 órdenes, 23 familias y 69 especies (Apéndice 2). Si se considera que el total de especies de mamíferos terrestres para México es de 477 (Arita y Ceballos, 1997), la REBIEN cuenta con el $15 \%$ del total nacional, y representa el 34\% del estado de Chipas, que cuenta con 204 especies (Retana y Lorenzo, 2001). Estos valores reflejan la importancia de la reserva (Figura 2).

De las especies registradas en la reserva, $12(17.3 \%)$ son las consideradas por la SEMARNAT, IUCN y CITES con algún tipo de protección (Apéndice 3). De las especies registradas en la reserva 12 (17\%) son consideradas bajo algún criterio de conservación por SEMARNAT, IUCN ó CITES (Apéndice 2). En cuanto a distribución, 41 especies (59.4\%) son compartidas con Sudamérica, lo que demuestra su afinidad hacia esta región. Se encuentran 17 especies (24.6 \%) endémicas de Mesoamérica; nueve (13\%) son compartidas con Norteamérica y Sudamérica lo que demuestra que esta reserva mantiene el flujo de especies entre diferentes regiones en ambos sentidos, retroalimentando la diversidad mastofaunística en el sur de México. Finalmente, una especie Glossophaga morenoi, es endémica de México. La población de "La Encrucijada" representa el límite de distribución al sur (Figura 3).

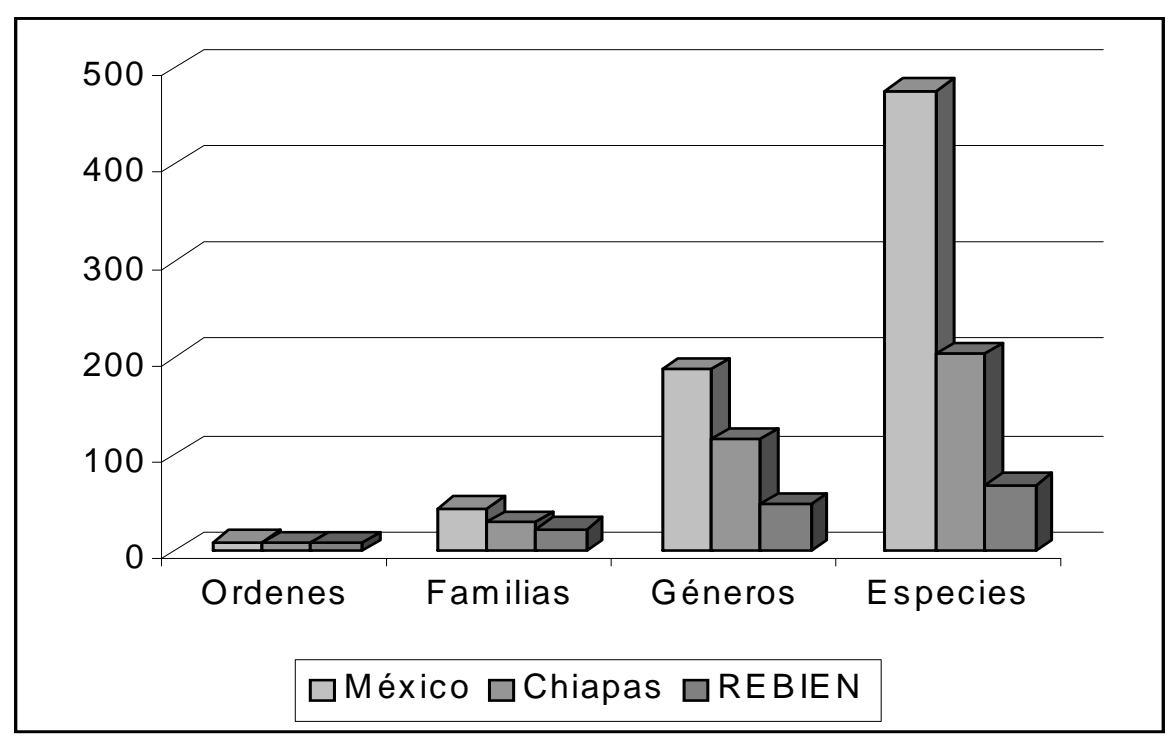

Figura 2. Número de órdenes, familias, géneros y especies de México, Chiapas y la REBIEN.. 
Existen 14 especies con importancia económica, ya que son utilizados como fuente de alimento, mascotas, o como adorno en forma de piel o disecada (Apéndice 3). Entre estos destacan por su importancia y vulnerabilidad Ateles geoffroyi, Leopardus pardalis, Tamandua mexicana y Dasyprocta punctata.

Existen varias especies de roedores y quirópteros de las cuales se desconocen aspectos de su historia natural. Estas conforman la mayor parte de las poblaciones de mamíferos, y son las que mantienen de manera general al ecosistema. Estas especies están sufriendo también el impacto, de las actividades del hombre; sin embargo no han sido tomadas en cuenta para una protección adecuada. La REBIEN es la única reserva en México que protege a algunas de estas especies como el murciélago Noctilio albiventris.

En el Estado de Chiapas se presentan dos corredores biológicos importantes para la distribución y dispersión de las especies entre Centroamérica y el norte de México. El primero se encuentra en el norte del estado con la selva Lacandona y la región Este, en donde predominan las selvas altas y medianas. El segundo corredor es la costa del Pacífico en donde se encuentran varios tipos de vegetación como selvas secas, manglares, pastizales y selvas altas y medianas, complementándose con la Sierra Madre, con mayor número de tipos de vegetación y gran variedad de climas por los cambios en el gradiente de humedad y altitud. En este sentido, "La Encrucijada"

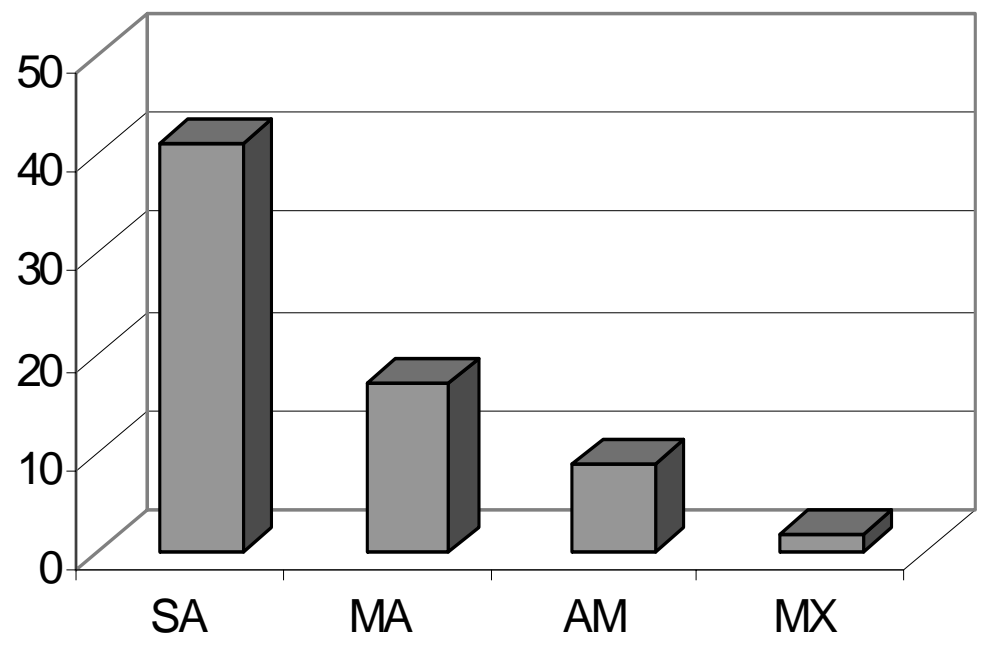

Figura 3. Total de especies de acuerdo a su distribución. SA (Compartidas con Sudamérica), MA (Mesoamericanas), AM (Americanas) y MX (Endémicas de México). 
representa un refugio único para especies que se encuentran amenazadas o en peligro de extinción, y el último corredor biológico en el pacífico en donde pueden mantener el flujo genético las poblaciones de mamíferos silvestres provenientes de Centro y Sudamérica.

\section{AGRADECIMIENTOS}

Agradecemos la colaboración del personal de vigilancia de la Reserva de la Biosfera La Encrucijada Sr. Efrén Montes y Carlos Hilerio Sánchez por su invaluable apoyo en campo y recopilación de la información. También agradecemos al Dr. Ricardo López_Wilchis, Dr. Eduardo Naranjo y Dra. Consuelo Lorenzo por la revisión y valiosos comentarios al manuscrito. A Alejandro Flamenco y Adrián Sarabia por su apoyo en el manejo de las bases de datos. Este proyecto fue parcialmente financiado por la Comisión Nacional para el Conocimiento y Uso de la Biodiversidad (CONABIO) clave P060.

\section{LITERATURA CITADA}

Álvarez, T., S. T. Álvarez y J.C. López-Vidal. 1994. Claves para murciélagos mexicanos. Centro de Investigaciones Biológicas del Noroeste e Instituto Politécnico Nacional, Escuela Nacional de Ciencias Biológicas. La Paz, Baja California Sur.

Arita, H. T. y G. Ceballos. 1997. Los mamíferos de México: distribución y estado de conservación. Revista Mexicana de Mastozoología, 2:33-71.

CITES. 1992. Convención sobre el comercio Internacional de Especies Amenazadas de Fauna y Flora Silvestres. Apéndices I y II. Washington, D.

Ceballos, G., J. Arroyo-Cabrales y R.A. Medellín. 2002. The Mammals of México: composition, distribution, and conservation status. Ocasional Papers Museum of Texas Tech University, 218:1-27.

Contreras, F. 1988. Las Lagunas Costeras Mexicanas. Centro de Ecodesarrollo, SEPESCA, México, D. F.

Contreras, F. 1993. Ecosistemas Costeros Mexicanos. CONABIO, UAM, México D. F.

García, E. 1973. Modificación al sistema de clasificación climática de Köppen. Instituto de Geografía, UNAM, México, D. F.

Hall, E. R. 1981. The mammals of North America. John Wiley, New York, USA. 1175 pp.

International Union for Conservation of Nature and Natural Resourses. 2003. IUCN Red List of Threatened animals. IUCN, Gland, Suiza.

Medellín, R. A., H. T. Arita y O. Sánchez. 1997. Identificación de los murciélagos de México, clave de campo. Asociación Mexicana de Mastozoología, A. C. México, D. F. 83 pp.

Reid, F. A. 1997. A field guide to the mammals of Central America and Southeast Mexico. Oxford University Press, New York.

Retana, O. G. y C. Lorenzo. 2001. Lista de los mamíferos terrestres de Chiapas: endemismos 
y estado de conservación. Acta Zoológica Mexicana, 85:25-49.

Secretaria de Medio Ambiente y Recursos Naturales. 2002. Norma Oficial Mexicana NOM059-ECOL-2002, Protección ambiental-Especies nativas de México de flora y fauna silvestres-Categorías de riesgo y especificaciones para su inclusión, exclusión o cambioLista de especies en riesgo. Diario Oficial de la Federación México. Segunda sección 1-85 pp.

Villa, R. B. 1966. Los Murciélagos de México. Instituto de Biología. Universidad Nacional Autónoma de México, D. F.

Wilson, D. E. y D.M. Reeder. 1993. Mammal species of the world. Segunda Edición. Smithsonian Institution Press, Washington, D.C. 


\section{APÉNDICE 1}

Colecciones con ejemplares de mamíferos de la REBIEN, Chiapas.

\begin{tabular}{lll}
\hline NUM & ACRÓNIMO & \\
\hline $\mathbf{1}$ & IHNMASTO & NOMBRE \\
$\mathbf{2}$ & UMMZ & Colección Zoológica Regional del Instituto de \\
$\mathbf{3}$ & LACM & University of Michigan Museum of Zoology \\
$\mathbf{4}$ & CMNH & Michigan. \\
$\mathbf{5}$ & ENCB & Los Angeles County Museum of Natural History. \\
& & Carnegie Museum of Natural History. \\
$\mathbf{6}$ & KU & Escuela Nacional de Ciencias Biológicas, Instituto \\
$\mathbf{7}$ & MZFC & Politécnico Nacional. \\
& & Kansas University, Museum of Zoology. \\
& & Museo de Zoología “Alfonso L. Herrera” de la \\
$\mathbf{1 0}$ & UTEP & Facultad de Ciencias de la Universidad Nacional \\
$\mathbf{9}$ & IBUNAM & Autónoma de México. \\
& & Colección Mastozoológica del Instituto de Biología \\
& & de la Universidad Nacional Autónoma de México. \\
& & University of California, Berkeley. Museum of \\
& & Uertebrate Zoology. \\
& & University of Texas at El Paso. Mammal Division.
\end{tabular}



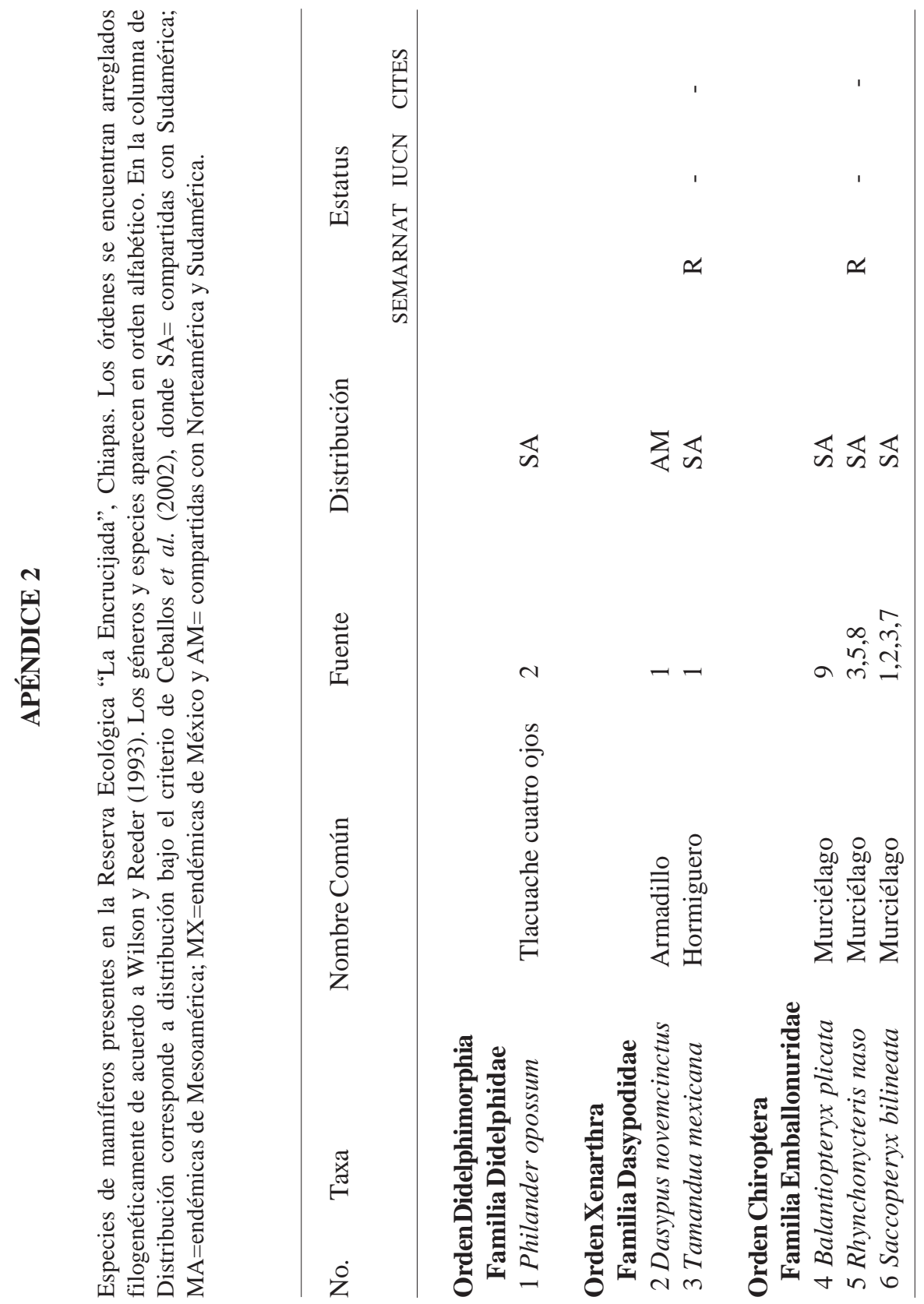


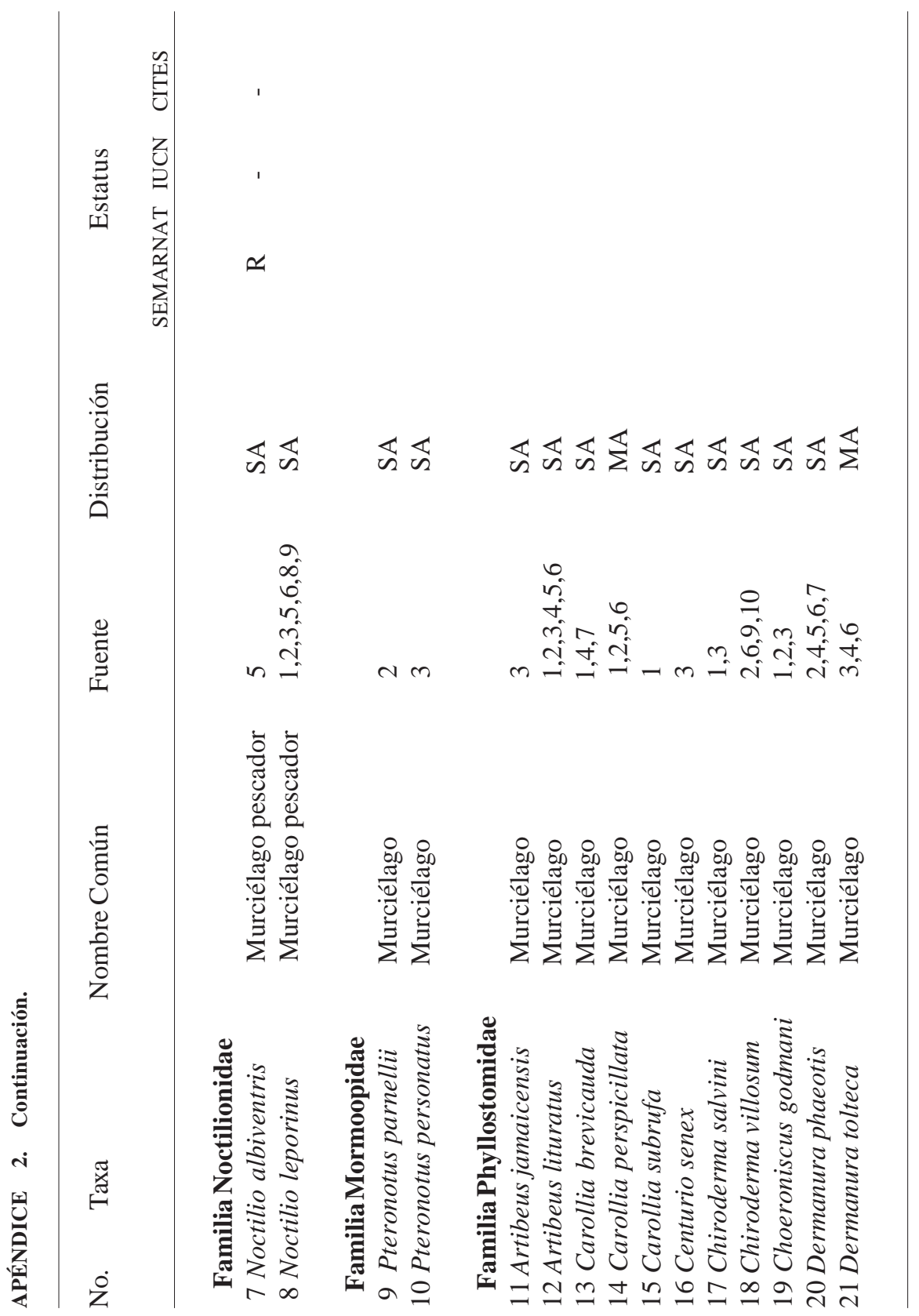




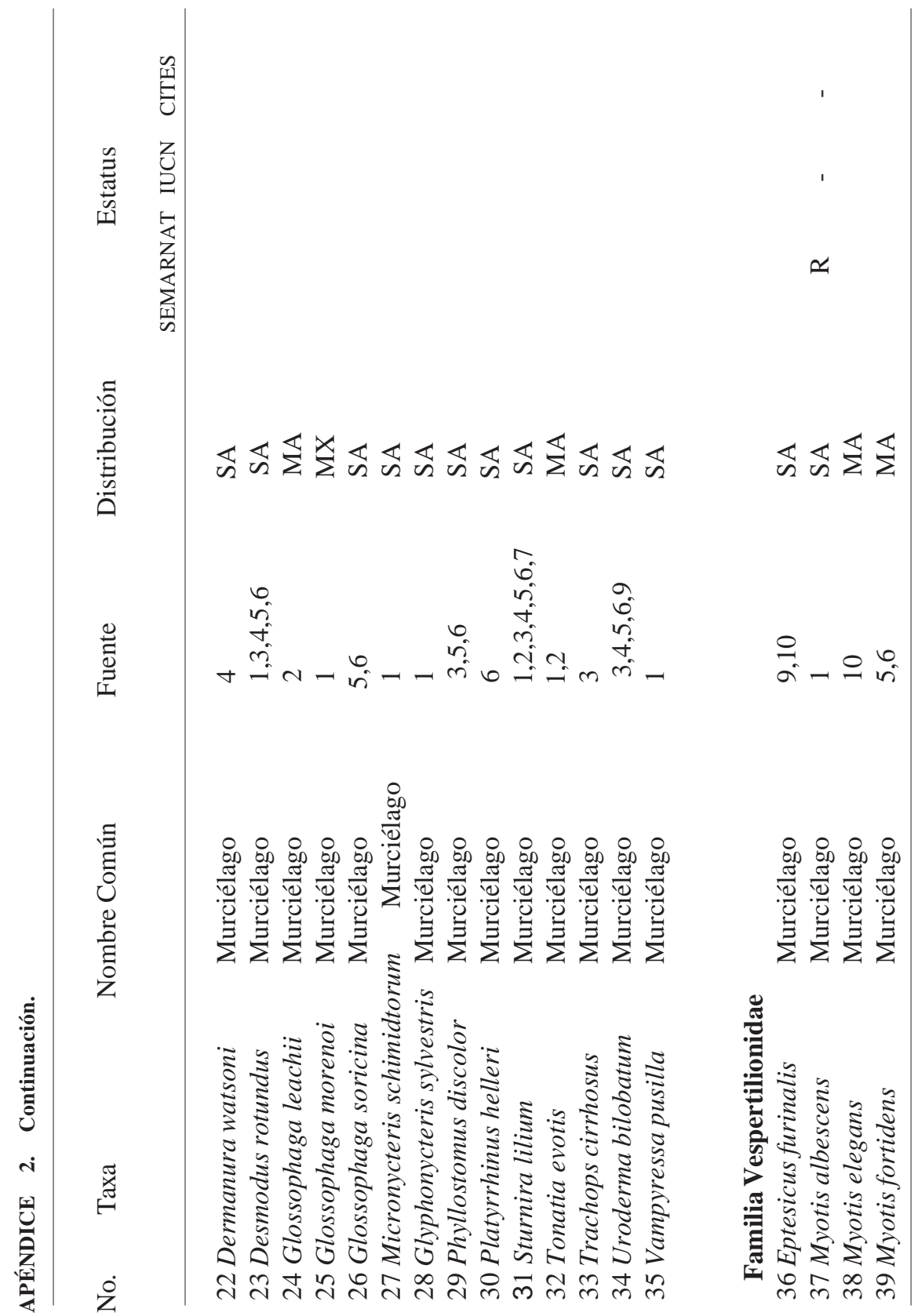




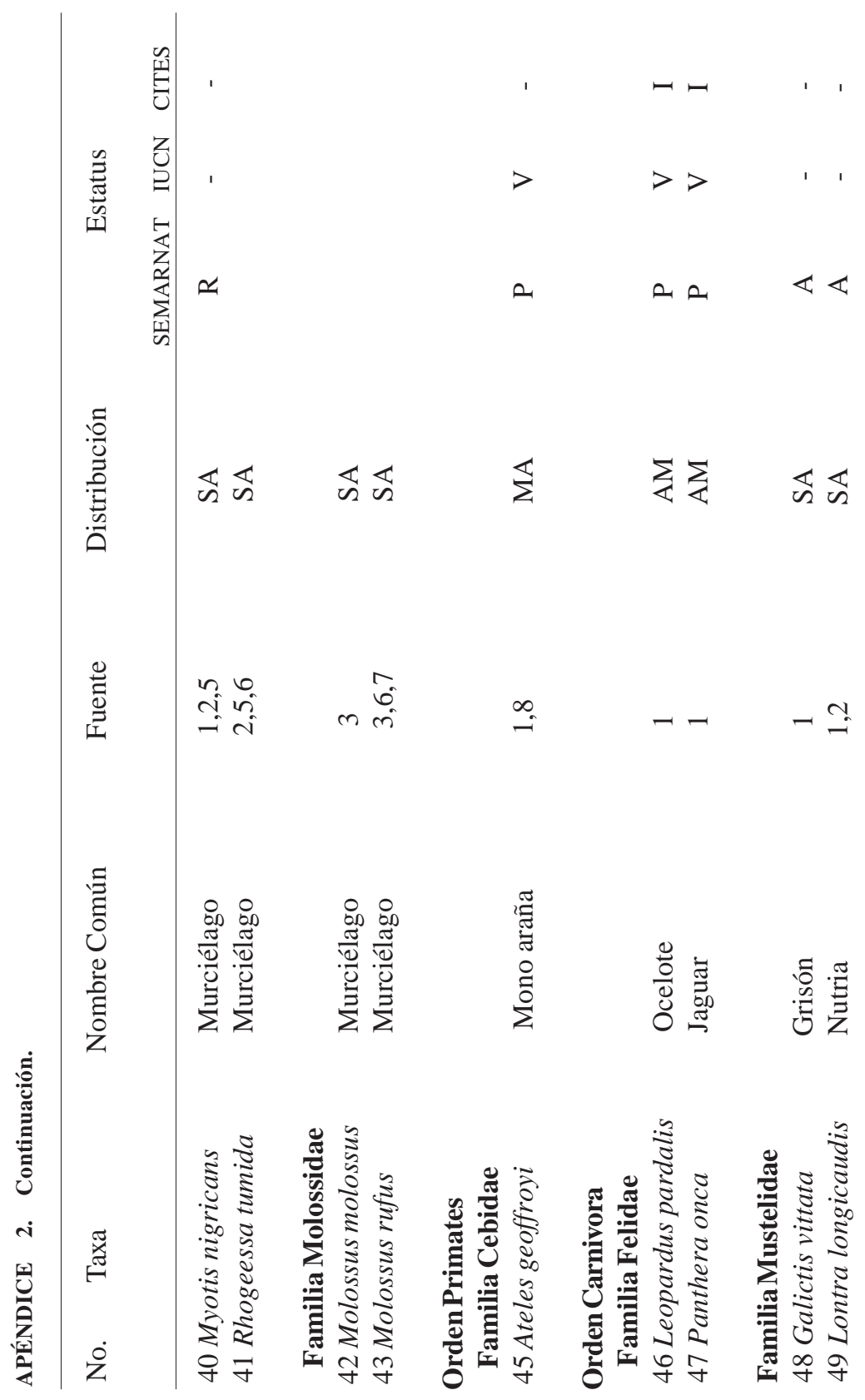




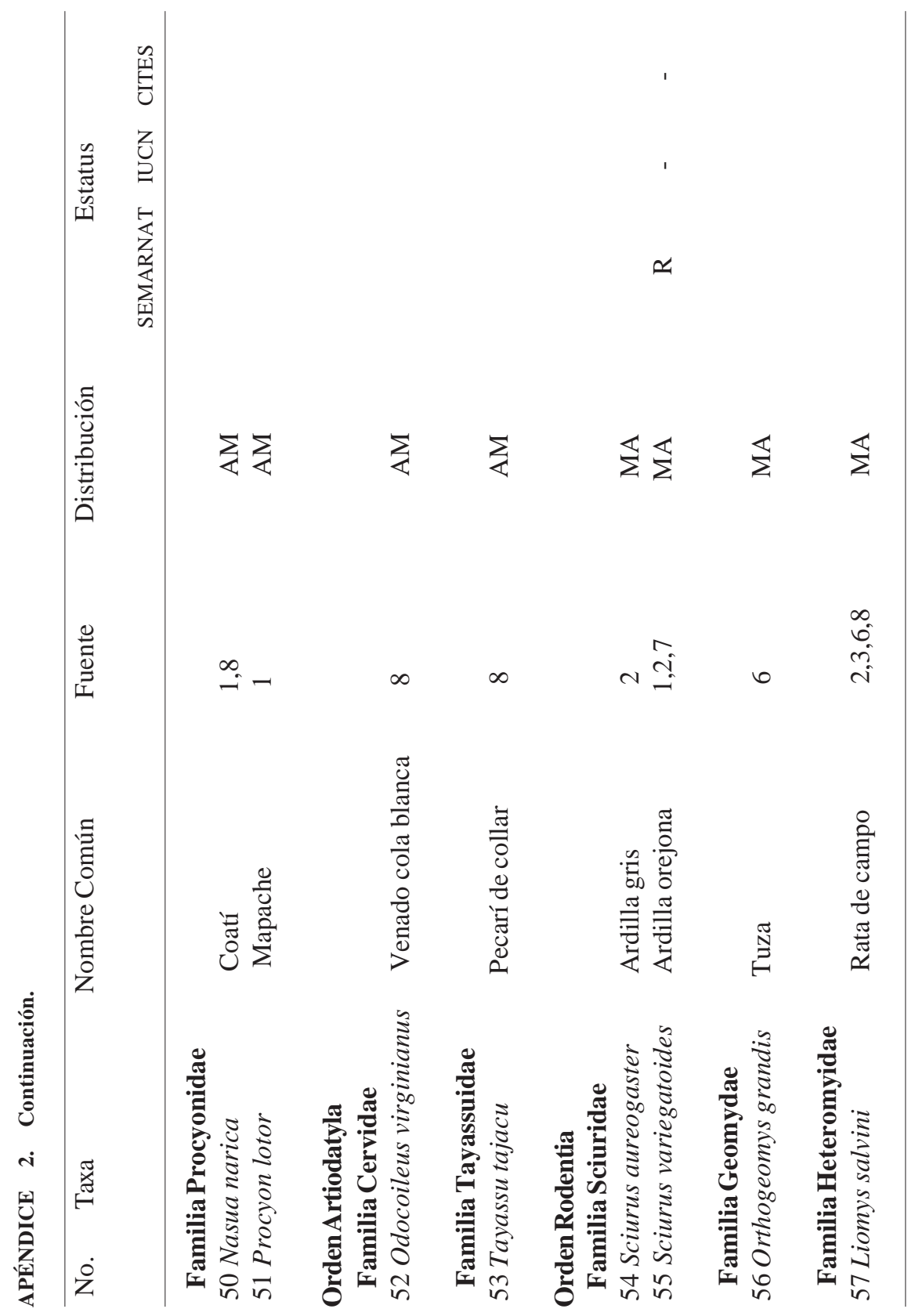




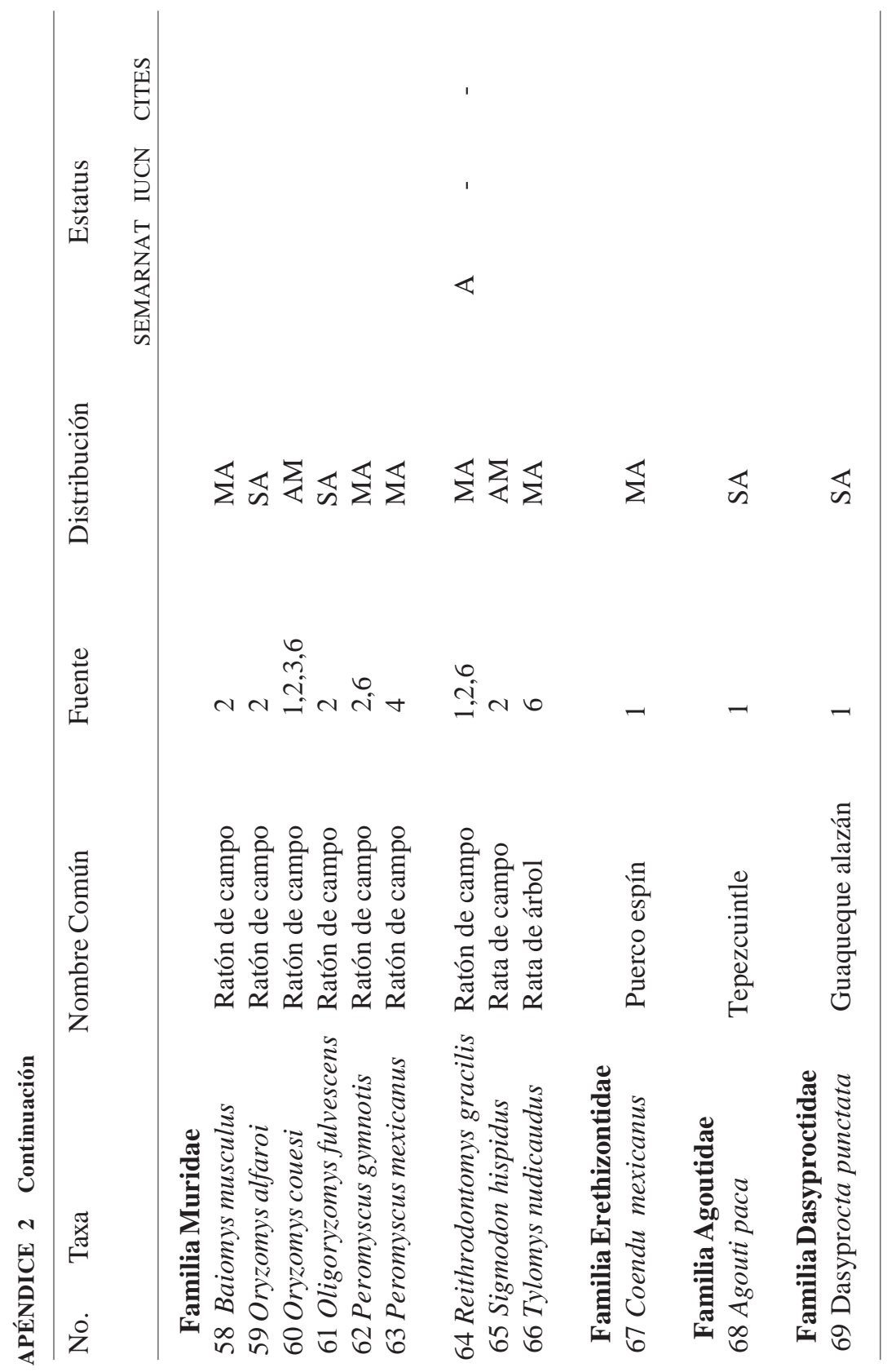




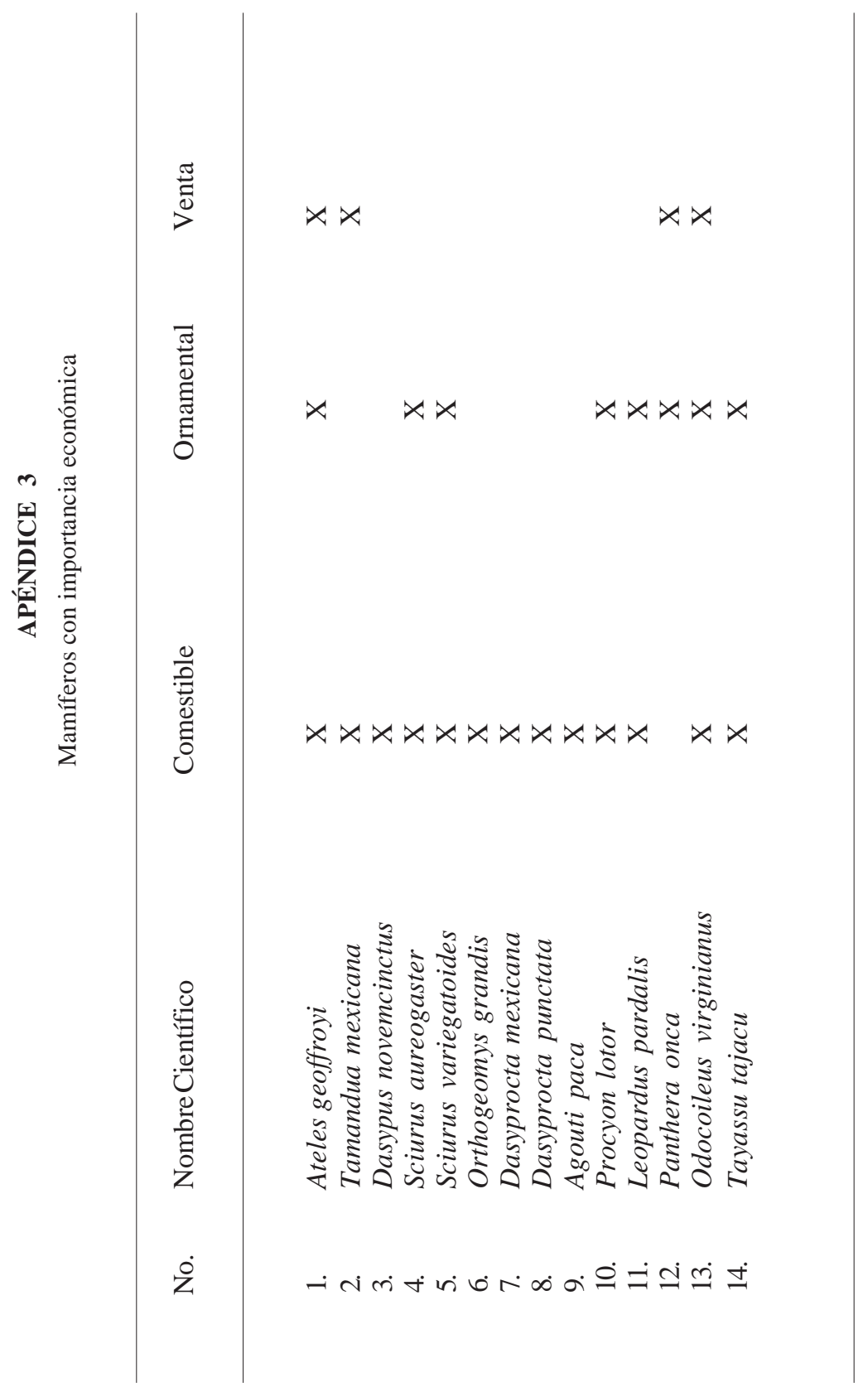

